\section{Cross-Eye Gain in Multi-Loop Retrodirective Cross-Eye Jamming}

Warren P. du Plessis, Senior Member, IEEE

\begin{abstract}
The simultaneous use of multiple retrodirective cross-eye jammers is analysed for both the case where the jammer loops point in different directions and when they point in the same direction. In both cases, the use of multiple cross-eye jammer loops is shown to lead to significantly increased angular errors in the threat radar under certain conditions. Alternatively, the sum-channel return can be increased to reduce the jammer-to-signal ratio (JSR) requirements for each jammer loop.
\end{abstract}

Index Terms-Cross-eye jamming, multi-loop cross-eye jamming, electronic warfare, and electronic countermeasures.

This work is based on the research supported in part by the Armaments Corporation of South Africa (Armscor), by the King Abdulaziz City for Science and Technology (KACST) and by the National Research Foundation of South Africa (NRF) (Grant specific unique reference number (UID) 85845). The NRF Grantholder acknowledges that opinions, findings and conclusions or recommendations expressed in any publication generated by the NRF supported research are that of the author(s), and that the NRF accepts no liability whatsoever in this regard.

Warren P. du Plessis is with the University of Pretoria, Pretoria, 0002, South Africa (e-mail:wduplessis@ieee.org).

\section{INTRODUCTION}

Multi-loop cross-eye jamming is the simultaneous use of multiple cross-eye jammer systems (loops) in an attempt to reduce both the tolerance sensitivity and jammer-to-signal ratio (JSR) requirements associated with cross-eye jamming [1]-[3]. These two issues constitute the main challenges associated with implementing a practical cross-eye jammer [4]-[10], so any improvement will justify the additional complexity associated with implementing multiple cross-eye jammer loops.

However, the retrodirective implementation of crosseye jamming has not been considered in published studies of multi-loop cross-eye jamming [1], [2]. This omission is significant because the retrodirective implementation appears to be the only practical way to realise operational cross-eye jammers [4]-[8], and previous work has shown that ignoring the retrodirective nature of a cross-eye jammer can lead to significant errors [10], [11].

This paper performs an analysis of multi-loop retrodirective cross-eye jamming. The focus is on the effect multiple retrodirective cross-eye jamming loops have on the cross-eye gain and indicated angle induced in the threat radar.

The case where the cross-eye jammer loops share a common centre (the concentric case) and the case where the cross-eye jammer loops are collinear are considered. In both cases, the use of multiple jammer loops can induce significantly larger errors in the threat radar, or alternatively, the sum-channel return can be increased to reduce the effect of platform skin return. To the best of the author's knowledge, neither of these possibilities have been considered previously in the open literature.

Multi-loop retrodirective cross-eye jamming is analysed in Section II, while the implications of this analysis are considered in Section III. The main results of this work are summarised in Section IV.

\section{ANALYSIS}

This section provides a mathematical analysis of multi-loop cross-eye jamming with the emphasis on the total cross-eye gain.

Note that, while the analysis below only explicity considers a phase-comparison monopulse radar, it has been shown that the results for this case are applicable to any monopulse radar [10], [12].

The geometry of a general two-loop cross-eye jammer system operating against a phase-comparison monopulse radar is shown in Fig. 1. 


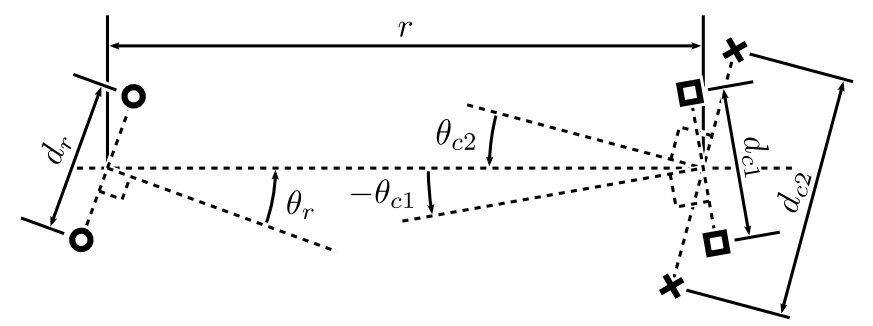

Fig. 1. The geometry of a multi-loop cross-eye jammer engagement where all jammer loops have the same range $r$. The phase centres of the radar, and the first and second jammer loops are denoted by circles, squares and crosses respectively.

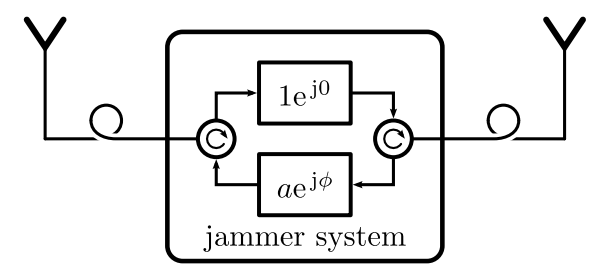

Fig. 2. The retrodirective implementation of a cross-eye jammer showing the definition of $a$ and $\phi$.

The sum- and difference channel returns from each cross-eye jammer loop are given by [10], [11]

$$
\begin{gathered}
S_{r n}=A_{n} \mathrm{e}^{\mathrm{j} \Phi_{n}} P_{a n}\left(\theta_{r}, \theta_{c n}, \theta_{e n}\right) \times \\
\frac{1}{2}\left(1+a_{n} \mathrm{e}^{\mathrm{j} \phi_{n}}\right)\left[\cos \left(2 k_{n}\right)+\cos \left(2 k_{c n}\right)\right] \\
D_{r n}=A_{n} \mathrm{e}^{\mathrm{j} \Phi_{n}} P_{a n}\left(\theta_{r}, \theta_{c n}, \theta_{e n}\right) \times \\
\mathrm{j} \frac{1}{2}\left[\left(1+a_{n} \mathrm{e}^{\mathrm{j} \phi_{n}}\right) \sin \left(2 k_{n}\right)+\right. \\
\left.\left(1-a_{n} \mathrm{e}^{\mathrm{j} \phi_{n}}\right) \sin \left(2 k_{c n}\right)\right]
\end{gathered}
$$

with

$$
\begin{aligned}
P_{a n}\left(\theta_{r}, \theta_{c n}, \theta_{e n}\right)= & P_{r}\left(\theta_{r}-\theta_{e n}\right) P_{r}\left(\theta_{r}+\theta_{e n}\right) \times \\
& P_{c n}\left(\theta_{c n}-\theta_{e n}\right) P_{c n}\left(\theta_{c n}+\theta_{e n}\right)
\end{aligned}
$$

$$
\begin{aligned}
k_{n} & =\beta \frac{d_{r}}{2} \sin \left(\theta_{r}\right) \cos \left(\theta_{e n}\right) \\
k & \approx \beta \frac{d_{r}}{2} \sin \left(\theta_{r}\right) \\
k_{c n} & =\beta \frac{d_{r}}{2} \cos \left(\theta_{r}\right) \sin \left(\theta_{e n}\right) \\
& \approx \beta \frac{d_{r}}{2} \cos \left(\theta_{r}\right) \theta_{e n}
\end{aligned}
$$

where the geometrical parameters are defined in Fig. 1 and the remainder of the parameters are $\theta_{e n}$ is half the angular separation of the $n^{\text {th }}$ jammer loop's antennas as seen by the radar and is given by

$$
\theta_{e n} \approx \frac{d_{c n}}{2 r} \cos \left(\theta_{c n}\right),
$$

$d_{c n}$ is the antenna spacing for jammer loop $n$ (the jammer baseline),

$\theta_{c n}$ is the rotation of the $n^{\text {th }}$ jammer loop's broadside direction from the radar,

$a_{n}$ and $\phi_{n}$ are the magnitude and phase of the one direction of the $n^{\text {th }}$ jammer loop relative to the other direction through the same jammer loop, respectively, as shown in Fig. 2,

$P_{r}(\theta)$ is the pattern of the antenna elements comprising the radar antenna,

$P_{c n}(\theta)$ are the patterns of the antennas of the $n^{\text {th }}$ jammer loops,

$\beta$ is the free-space phase constant, and

$d_{r}$ is the separation of the radar antenna-element phase centres.

The approximations in (5), (7) and (8) are extremely accurate for practical cross-eye jamming scenarios where $r \gg d_{c n}$ [10], [11], [13].

The total sum- and difference-channel returns when all loops are considered are the sums of (1) and (2) respectively over all values of $n$ giving a monopulse ratio of [14]

$$
M_{M}=\Im\left\{\frac{\sum_{n=1}^{N} D_{r n}}{\sum_{n=1}^{N} S_{r n}}\right\}
$$

for $N$ jammer loops under the assumption of an exact monopulse processor. The indicated angle is computed from the monopulse ratio using [14]

$$
\begin{aligned}
M_{M} & =\tan \left[\beta \frac{d_{r}}{2} \sin \left(\theta_{i}\right)\right] \\
& \approx \tan \left(k_{i}\right)
\end{aligned}
$$

where $\theta_{i}$ is the monopulse indicated angle and $k_{i}$ is the value of (4) with $\theta_{r}=\theta_{i}$ and where $\theta_{e}$ is small enough to assume that $\cos \left(\theta_{e}\right) \approx 1$. The approximation in (11) is accurate when $\theta_{e}$ is small [13], [15].

The main difference to the previously-published single-loop retrodirective cross-eye analyses [9], [10], [16] is the inclusion of the factor $A_{n} \mathrm{e}^{\mathrm{j} \Phi_{n}}$ in (1) and (2) to account for differences between the jammer loops. These factors include the effects of path-length differences, component differences and differences as a result of the action of the jammer. 
Using (1) and (2) to expand the monopulse ratio in (9) leads to

$$
\begin{aligned}
& M_{M}=\Re\left\{\frac{\sum_{n=1}^{N} P_{a n}\left(\theta_{r}, \theta_{c n}, \theta_{e n}\right) A_{n} \mathrm{e}^{\mathrm{j} \Phi_{n}} \times \cdots}{\sum_{n=1}^{N} P_{a n}\left(\theta_{r}, \theta_{c n}, \theta_{e n}\right) A_{n} \mathrm{e}^{\mathrm{j} \Phi_{n}} \times \cdots}\right. \\
& \frac{\cdots\left[\left(1+a_{n} \mathrm{e}^{\mathrm{j} \phi_{n}}\right) \sin (2 k)+\cdots\right.}{\cdots\left(1+a_{n} \mathrm{e}^{\mathrm{j} \phi_{n}}\right) \times \cdots} \\
& \left.\frac{\left.\cdots\left(1-a_{n} \mathrm{e}^{\mathrm{j} \phi_{n}}\right) \sin \left(2 k_{c n}\right)\right]}{\cdots\left[\cos (2 k)+\cos \left(2 k_{c n}\right)\right]}\right\} \\
& \approx \Re\left\{\frac{\sum_{n=1}^{N} P_{a n}\left(\theta_{r}, \theta_{c n}, \theta_{e n}\right) A_{n} \mathrm{e}^{\mathrm{j} \Phi_{n}} \times \cdots}{\sum_{n=1}^{N} P_{a n}\left(\theta_{r}, \theta_{c n}, \theta_{e n}\right) A_{n} \mathrm{e}^{\mathrm{j} \Phi_{n}} \times \cdots}\right. \\
& \left.\frac{\cdots\left(1+a_{n} \mathrm{e}^{\mathrm{j} \phi_{n}}\right)}{\cdots\left(1+a_{n} \mathrm{e}^{\mathrm{j} \phi_{n}}\right)}\right\} \frac{\sin (2 k)}{\cos (2 k)+1}+ \\
& \Re\left\{\frac{\sum_{n=1}^{N} P_{a n}\left(\theta_{r}, \theta_{c n}, \theta_{e n}\right) A_{n} \mathrm{e}^{\mathrm{j} \Phi_{n}} \times \cdots}{\sum_{n=1}^{N} P_{a n}\left(\theta_{r}, \theta_{c n}, \theta_{e n}\right) A_{n} \mathrm{e}^{\mathrm{j} \Phi_{n}} \times \cdots}\right. \\
& \left.\frac{\cdots\left(1-a_{n} \mathrm{e}^{\mathrm{j} \phi_{n}}\right) \sin \left(2 k_{c n}\right)}{\cdots\left(1+a_{n} \mathrm{e}^{\mathrm{j} \phi_{n}}\right)[\cos (2 k)+1]}\right\} \\
& =\tan (k)+\frac{1}{2 \cos ^{2}(k)} \times \\
& \Re\left\{\begin{array}{l}
\sum_{n=1}^{N} P_{a n}\left(\theta_{r}, \theta_{c n}, \theta_{e n}\right) A_{n} \mathrm{e}^{\mathrm{j} \Phi_{n}} \times \cdots \\
\sum_{n=1}^{N} P_{a n}\left(\theta_{r}, \theta_{c n}, \theta_{e n}\right) A_{n} \mathrm{e}^{\mathrm{j} \Phi_{n}} \times \cdots
\end{array}\right. \\
& \left.\frac{\cdots\left(1-a_{n} \mathrm{e}^{\mathrm{j} \phi_{n}}\right) \sin \left(2 k_{c n}\right)}{\cdots\left(1+a_{n} \mathrm{e}^{\mathrm{j} \phi_{n}}\right)}\right\}
\end{aligned}
$$

where the accurate assumption $\cos \left(2 k_{c n}\right) \approx 1$ [13] was used to simplify the result.

The effect of the radar and jammer antenna patterns in (14) does not cancel as it does in the single-loop case [10], [11]. This is due to the assumption that each jammer loop can have a different rotation $\left(\theta_{c n}\right)$ and baseline $\left(d_{c n}\right)$. Nevertheless, some simplifications are possible.

Given that the properties of a phase-comparison monopulse antenna are predominantly determined by the separation of the two radar antenna elements [14], the patterns of the radar antenna elements $\left(P_{r}(\theta)\right)$ can be expected to vary only slowly with angle. Specifically, variations over angular range of the jammer antennas $\left(\theta_{r}-\theta_{e n}\right.$ to $\left.\theta_{r}+\theta_{e n}\right)$ will be assumed to be negligible

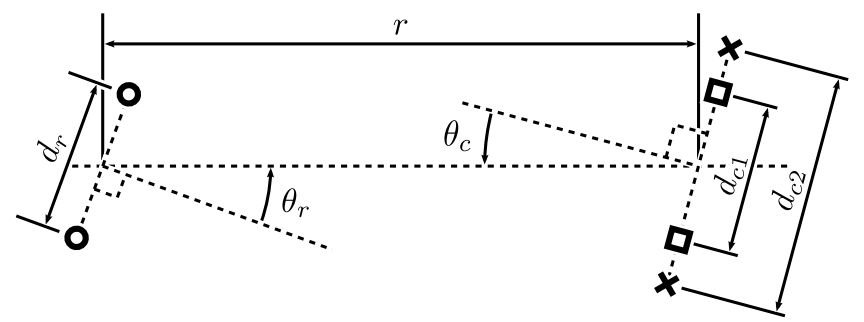

Fig. 3. The change to the geometry in Fig. 1 to ensure that all jammer loops have the same rotation $\theta_{c}$.

leading to

$$
P_{r}\left(\theta_{r}-\theta_{e n}\right) \approx P_{r}\left(\theta_{r}+\theta_{e n}\right) \approx P_{r}\left(\theta_{r}\right)
$$

to a high degree of accuracy, especially near the radar's boresight direction [13].

A similar argument can be applied to the jammer antennas to obtain

$$
P_{c n}\left(\theta_{c n}-\theta_{e n}\right) \approx P_{c n}\left(\theta_{c n}+\theta_{e n}\right) \approx P_{c n}\left(\theta_{c n}\right)
$$

and when the jammer antennas are identical and collinear as shown in Fig. 3,

$$
P_{c n}\left(\theta_{c n}\right) \approx P_{c}\left(\theta_{c}\right) .
$$

The simplifications for the jammer antennas should be more accurate than for the radar antenna elements in (15) as jammer antennas either have wide beamwidths to achieve large angular coverage or have their main beams pointed towards the threat radar if phased arrays are used. The form of the antenna factor in (3) can thus be simplified to

$$
P_{a n}\left(\theta_{r}, \theta_{c n}, \theta_{e n}\right) \approx\left[P_{r}\left(\theta_{r}\right) P_{c n}\left(\theta_{c n}\right)\right]^{2} .
$$

The radar antenna-element patterns cancel in the monopulse ratio $\left(M_{M}\right)$ as the $P_{r}\left(\theta_{r}\right)$ factors appear in both the numerator and denominator of (14). The jammer-antenna patterns cancel if the jammer antennas are identical and collinear, and as described above, the reasonable approximations in (16) can be used to simplify the form of the monopulse ratio $\left(M_{M}\right)$.

Defining the jammer-baseline ratio as

$$
d_{c r n}=\frac{d_{c n}}{d_{c N}}
$$

gives

$$
\begin{aligned}
\sin \left(2 k_{c n}\right) & \approx 2 k_{c n} \\
& \approx \beta d_{r} \cos \left(\theta_{r}\right) \theta_{e n} \\
& =\beta d_{r} \cos \left(\theta_{r}\right) \frac{d_{c N}}{2 r} \cos \left(\theta_{c n}\right) d_{c r n}
\end{aligned}
$$


where $\sin \left(2 k_{c n}\right) \approx 2 k_{c n}$ was assumed in light of the fact that $\cos \left(2 k_{c n}\right) \approx 1$ has already been assumed. This result can be further simplified to

$$
\sin \left(2 k_{c n}\right) \approx 2 k_{c N} d_{c r n}
$$

when the jammer antennas are collinear because all the jammer rotations $\left(\theta_{c n}\right)$ are identical.

Equation (14) can now be rewritten as

$$
\begin{aligned}
& M_{M} \approx \tan (k)+\frac{\beta d_{r} \cos \left(\theta_{r}\right) \frac{d_{c}}{2 r}}{2 \cos ^{2}(k)} \times \\
& \Re\left\{\begin{array}{l}
\frac{\sum_{n=1}^{N}\left[P_{c n}\left(\theta_{c n}\right)\right]^{2} A_{n} \mathrm{e}^{\mathrm{j} \Phi_{n}} \times \cdots}{\sum_{n=1}^{N}\left[P_{c n}\left(\theta_{c n}\right)\right]^{2} A_{n} \mathrm{e}^{\mathrm{j} \Phi_{n}} \times \cdots} \\
\left.\frac{\cdots\left(1-a_{n} \mathrm{e}^{\mathrm{j} \phi_{n}}\right) \cos \left(\theta_{c n}\right) d_{c r n}}{\cdots\left(1+a_{n} \mathrm{e}^{\mathrm{j} \phi_{n}}\right)}\right\}
\end{array}\right.
\end{aligned}
$$

which reduces to

$$
\begin{aligned}
& M_{M c} \approx \tan (k)+\frac{k_{c}}{\cos ^{2}(k)} \times \\
& \Re\left\{\frac{\sum_{n=1}^{N} A_{n} \mathrm{e}^{\mathrm{j} \Phi_{n}}\left(1-a_{n} \mathrm{e}^{\mathrm{j} \phi_{n}}\right) d_{c r n}}{\sum_{n=1}^{N} A_{n} \mathrm{e}^{\mathrm{j} \Phi_{n}}\left(1+a_{n} \mathrm{e}^{\mathrm{j} \phi_{n}}\right)}\right\}
\end{aligned}
$$

when the jammer antennas are identical and collinear. Jammer loop $N$ is assumed to have the largest baseline, so $d_{c N}=d_{c}$ and $k_{c N}=k_{c}$ are used to allow comparisons to a single jammer loop.

The factors after the first lines of (24) and (25) fulfil the same role as that of the cross-eye gain. The cross-eye gain equivalent for a multi-loop retrodirective cross-eye jammer is

$$
\begin{aligned}
& G_{C M}=\Re\left\{\frac{\sum_{n=1}^{N}\left[P_{c n}\left(\theta_{c n}\right)\right]^{2} A_{n} \mathrm{e}^{\mathrm{j} \Phi_{n}} \times \cdots}{\sum_{n=1}^{N}\left[P_{c n}\left(\theta_{c n}\right)\right]^{2} A_{n} \mathrm{e}^{\mathrm{j} \Phi_{n}} \times \cdots}\right. \\
& \left.\frac{\cdots\left(1-a_{n} \mathrm{e}^{\mathrm{j} \phi_{n}}\right) \cos \left(\theta_{c n}\right) d_{c r n}}{\cdots\left(1+a_{n} \mathrm{e}^{\mathrm{j} \phi_{n}}\right)}\right\}
\end{aligned}
$$

where the inclusion of $\cos \left(\theta_{c n}\right)$ means that $G_{C M}$ is not a true cross-eye gain because the monopulse ratio in (24) does not reduce to the same form as for a single-loop retrodirective cross-eye jammer.

The cross-eye gain for the collinear case is given by

$$
G_{C M c}=\Re\left\{\frac{\sum_{n=1}^{N} A_{n} \mathrm{e}^{\mathrm{j} \Phi_{n}}\left(1-a_{n} \mathrm{e}^{\mathrm{j} \phi_{n}}\right) d_{c r n}}{\sum_{n=1}^{N} A_{n} \mathrm{e}^{\mathrm{j} \Phi_{n}}\left(1+a_{n} \mathrm{e}^{\mathrm{j} \phi_{n}}\right)}\right\}
$$

and this is a true cross-eye gain as the form of the monopulse ratio can be rewritten as

$$
\begin{aligned}
M_{M c} & \approx \tan (k)+\frac{k_{c}}{\cos ^{2}(k)} G_{C M c} \\
& \approx \frac{\sin (2 k)+\sin \left(2 k_{c}\right) G_{C M c}}{\cos (2 k)+\cos \left(2 k_{c}\right)}
\end{aligned}
$$

which is identical to the monopulse ratio of a single-loop retrodirective cross-eye jammer [10], [11] apart from the different cross-eye gain.

\section{DISCUSSION}

The implications of the results derived in Section II are considered below. The special case of two-loop retrodirective cross-eye jammers will be considered to simplify the analysis without loss of generality.

The following parameters typical of a missile threat against an aircraft or ship [10], [11] will be used unless otherwise specified:

- $10 \mathrm{GHz}$ radar frequency,

- $10^{\circ}$ radar beamwidth $\left(d_{r}=2.54\right.$ wavelengths, and each radar antenna element is an equally-excited linear source 2.54 wavelengths long),

- $1 \mathrm{~km}$ jammer range $(r=1 \mathrm{~km})$,

- $10 \mathrm{~m}$ larger jammer baseline $\left(d_{c 2}=10 \mathrm{~m}\right)$,

- $5 \mathrm{~m}$ smaller jammer baseline $\left(d_{c 1}=5 \mathrm{~m}\right)$,

- $30^{\circ}$ jammer direction offset or collinear jammer loops $\left(\theta_{2}-\theta_{1}=30^{\circ}\right.$ or $\left.0^{\circ}\right)$,

- jammer antennas with a $60^{\circ}$ beamwidth $\left(P_{c n}(\theta)=\right.$ $\cos (0.75 \theta))$,

- $0.5 \mathrm{~dB}$ jammer amplitude mismatch $\left(a_{n}=0.9441\right)$ for all jammer loops, and

- the relative phases of the two directions through all the jammer loops are $180^{\circ}\left(\phi_{n}=180^{\circ}\right)$.

The 3-dB beamwidths and first nulls of the radar sum channel are indicated by " $3 \mathrm{~dB}$ " and "Null" on the right axis of the indicated-angle plots below, and the broadside directions of the jammer loops are indicated on the top axis, where appropriate.

\section{A. Concentric Jammer Loops}

The main application of concentric retrodirective cross-eye jammer loops which are not collinear (Fig. 1) is to extend the angular range over which a cross-eye jammer system is effective.

Fig. 4 shows a number of cases of concentric multiloop retrodirective cross-eye jammers with the smaller baseline being $5 \mathrm{~m}$ or $10 \mathrm{~m}$. The first case ("Single 2") considers the loop with its boresight at $0^{\circ}$ alone, while the second two cases ("Single 1") consider the loop with its boresight at $30^{\circ}$ alone for two different baselines $(5 \mathrm{~m}$ 

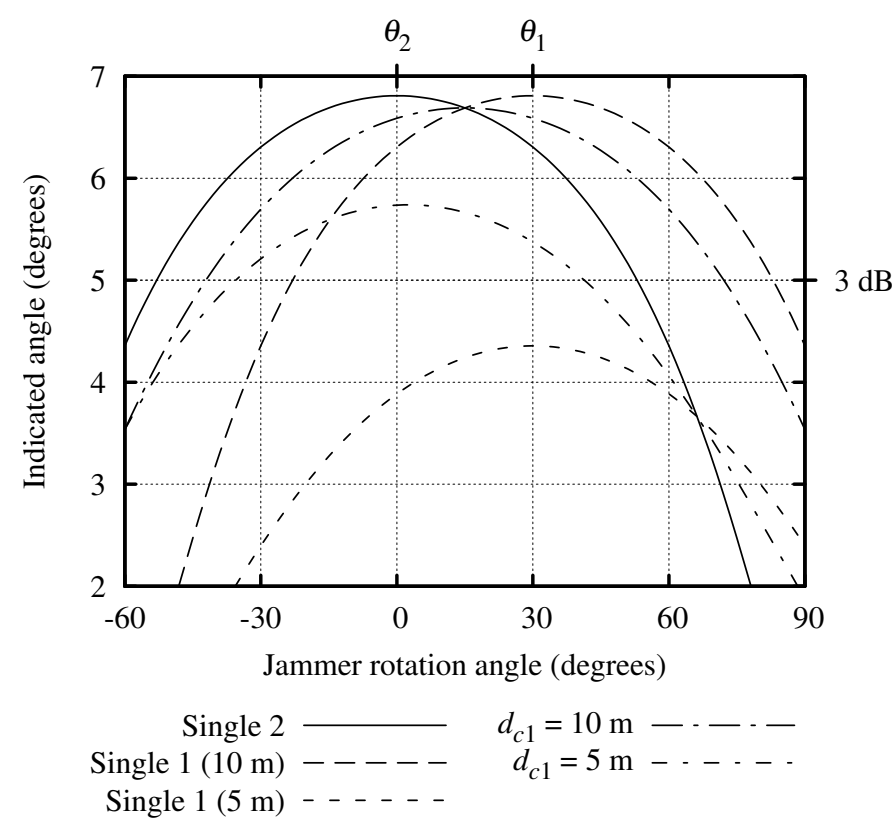

Fig. 4. The indicated angle for identical jammer loops which have an angular offset of $30^{\circ}$ for the cases described in the text.

and $10 \mathrm{~m}$ ). The last two cases consider the two jammer loops operating together with two different baselines for the loop with its boresight at $30^{\circ}$. The two-loop cases assume that the jammer loops are identical $\left(A_{1}=A_{2}\right.$ and $\Phi_{2}-\Phi_{1}=0$ ).

The conclusion from Fig. 4 is that the cross-eye gain equivalent for the two jammer loops operating together is always lower than for one of the jammer loops operating in isolation when the two loops are identical. There is thus no reason to use multiple identical retrodirective cross-eye jammer loops to improve angular coverage.

However, the situation changes dramatically when the returns from the two jammer loops are not identical as shown in Fig. 5 where the jammer-loop amplitudes differ by $0.5 \mathrm{~dB}\left(A_{2} / A_{1}=0.5 \mathrm{~dB}\right)$ and have a $180^{\circ}$ phase difference $\left(\Phi_{2}-\Phi_{1}=180^{\circ}\right)$. The indicatedangle magnitudes (and thus the cross-eye gain equivalent magnitudes) are significantly greater than the single-loop values from Fig. 4 for most jammer rotations in Fig. 5.

The reason for the increase in the indicated angle is the fact that the magnitude of the denominator of the crosseye gain equivalent in (26) is reduced because its terms have a phase difference of $180^{\circ}$. In the case considered, the denominator of (26) is zero approximately halfway between the two jammer-loop broadside directions, explaining the sudden changes of the signs of the indicated angles at this point in Fig. 5.

Limiting the apparent target from a cross-eye jammer to one side of the jammer is desirable as outlined in [15]. However, as shown in Fig. 5, this does not occur with

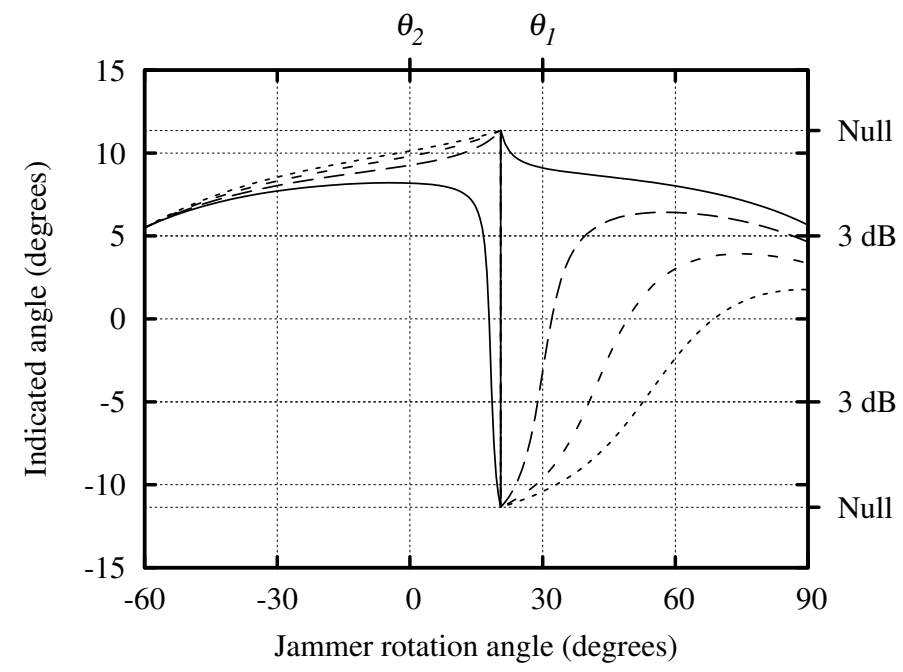

$$
\begin{gathered}
d_{c 1}=10 \mathrm{~m}-\quad \begin{array}{r}
d_{c 1}=5 \mathrm{~m} \ldots \\
d_{c 1}=7.5 \mathrm{~m}-----
\end{array} \quad d_{c 1}=2.5 \mathrm{~m} \ldots \ldots
\end{gathered}
$$

Fig. 5. The indicated angle for identical jammer loops which have an angular offset of $30^{\circ}$ for the case described in the text.

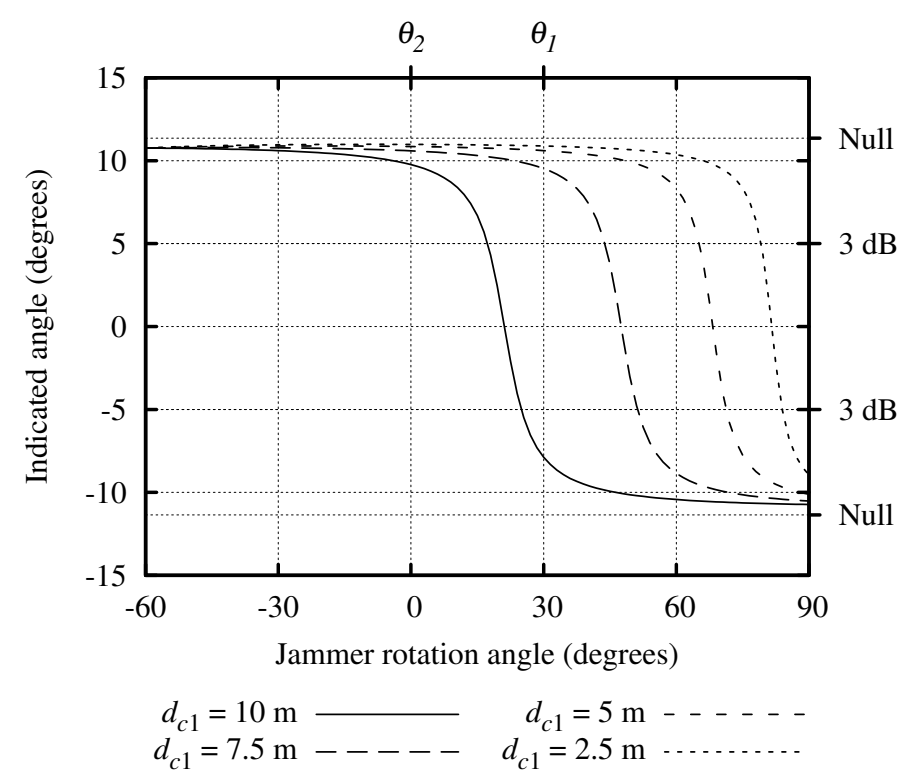

Fig. 6. The indicated angle for identical jammer loops which have an angular offset of $30^{\circ}$ for the case considered in the text.

directional jammer antennas when the jammer loops have phase differences of $180^{\circ}$, so this case is thus unlikely to have practical application.

Noting that the denominator of the cross-eye gain equivalent in (26) has its smallest magnitude approximately halfway between the jammer-loop broadside directions largely as a result of the jammer-antenna patterns suggests that using omnidirectional jammer antennas will lead to larger indicated angles over the whole angular range. This possibility is explored in Fig. 6 where the jammer-loop amplitudes differ by $0.5 \mathrm{~dB}$ $\left(A_{2} / A_{1}=0.5 \mathrm{~dB}\right)$ and have a $180^{\circ}$ phase difference 
$\left(\Phi_{2}-\Phi_{1}=180^{\circ}\right)$.

The indicated angle magnitude is seen to be extremely high in Fig. 6 as a result of the cancellation of the two signals in the denominator of the cross-eye gain equivalent in (26). However, a large indicated angle is not achieved at all angles as the indicated angle becomes zero at those angles where the difference-channel return is zero. The jammer-rotation angle where the indicated angle becomes zero is seen to depend strongly on the jammer baselines.

Decreasing the smaller jammer baseline moves the angle where the indicated angle is zero further away from the broadside directions of the jammer loops. This is because the effect of a jammer loop on the numerator of the cross-eye gain equivalent in (26) decreases as the baseline decreases, while the effect on the denominator of (26) remains unchanged. The desired effect of the smaller jammer loop is thus to cancel the sum-channel return of the larger jammer loop without affecting the difference-channel return. The one drawback of cancellation in the denominator of the cross-eye gain equivalent in (26) is that this also decreases the sum-channel return from the jammer. The skin return from the platform mounting the cross-eye jammer will thus have an increased effect on the indicated angle as the system JSR decreases [13], [15].

The possibility of increasing the total sum-channel return to reduce the effect of platform skin return also exists. While this will reduce the cross-eye gain equivalent, the ability to produce a stable target in the presence of platform skin return is desirable. For example, when jammer loops are identical $\left(A_{n}=A\right.$ and $\left.\Phi_{n}=\Phi\right)$, the total sum-channel return as a result of all the crosseye jammer loops will be increased by $6 \mathrm{~dB}$, while the indicated angle will be comparable to that of a single jammer loop as seen from Fig. 4. The use of multiple retrodirective cross-eye jammer loops thus allows the possibility of a reduction of the JSR of the repeaters for each jammer loop shown in Fig .2.

\section{B. Collinear Jammer Loops}

Collinear retrodirective cross-eye jammer loops seek to improve the performance of a cross-eye jammer. Fig. 7 compares the indicated angle of single and multiple collinear jammer loops.

The total cross-eye gain in Fig. 7 is seen to increase as the baseline of the narrower loop increases. This result is anticipated as the terms in the numerator of the crosseye gain in (27) are scaled by the ratio of the baseline to the largest baseline. Using the largest possible baselines for all jammer loops thus leads to the largest indicated angle.

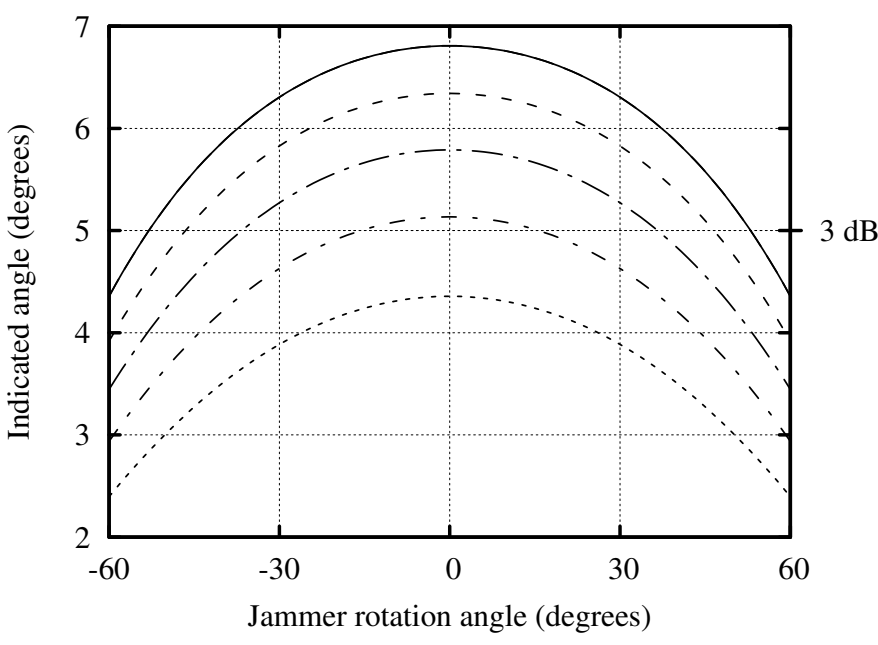

$$
\begin{array}{rr}
\text { Single } 2 & \text { Single } 1(5 \mathrm{~m}) \\
d_{c 1}=10 \mathrm{~m}-\ldots \ldots & \ldots . \\
d_{c 1}=7.5 \mathrm{~m}-\ldots- & d_{c 1}=5 \mathrm{~m}-\ldots- \\
& d_{c 1}=2.5 \mathrm{~m} \ldots \ldots
\end{array}
$$

Fig. 7. The indicated angle for identical collinear jammer loops. Note that the "Single 2" and " $d_{c 1}=10 \mathrm{~m}$ " curves are identical.

Fig. 7 also shows that single jammer loop with a $10 \mathrm{~m}$ baseline and two jammer loops with baselines of $10 \mathrm{~m}$ produce identical indicated-angle results. This outcome is anticipated in light of the form of the cross-eye gain in (27) which can be rewritten as

$$
\begin{aligned}
G_{C M c} & =d_{c r N} \Re\left\{\frac{1-a \mathrm{e}^{\mathrm{j} \phi}}{1+a \mathrm{e}^{\mathrm{j} \phi}} \times \frac{\sum_{n=1}^{N} A_{n} \mathrm{e}^{\mathrm{j} \Phi_{n}}}{\sum_{n=1}^{N} A_{n} \mathrm{e}^{\mathrm{j} \Phi_{n}}}\right\} \\
& =\Re\left\{\frac{1-a \mathrm{e}^{\mathrm{j} \phi}}{1+a \mathrm{e}^{\mathrm{j} \phi}}\right\} \\
& =G_{C}
\end{aligned}
$$

which is the cross-eye gain of a single retrodirective cross-eye jammer loop.

Perhaps more remarkably, the above result shows that the amplitude and phase shift between otherwise identical jammers $\left(A_{n}\right.$ and $\left.\Phi_{n}\right)$ does not affect the cross-eye gain. This promising result suggests that the matching between identical retrodirective cross-eye jammer loops is not crucial to system performance.

However, the effect of the jammer-loop matching is not completely arbitrary as it strongly affects the magnitude of the sum-channel return. For example, a perfectly matched dual-loop cross-eye jammer $\left(A_{n}=1\right.$ and $\Phi_{n}=\Phi$ ) leads to a sum-channel return which is $6 \mathrm{~dB}$ greater than the single-loop case, while setting $\sum A_{n} \mathrm{e}^{\mathrm{j} \Phi_{n}}=0$ leads to a zero sum-channel return. Multi-loop cross-eye jamming can thus lead to reduced JSR requirements in the presence of platform skin return. 
Ensure that the jammer loops are identical $\left(A_{n}=1\right.$ and $\left.\Phi_{n}=\Phi\right)$ means that one of the directions through each of the jammer loops is identical to the same path through the other jammer loops. This scenario gives a cross-eye gain of

$$
\begin{aligned}
G_{C M c} & =\Re\left\{\frac{\sum_{n=1}^{N}\left(1-a_{n} \mathrm{e}^{\mathrm{j} \phi_{n}}\right) d_{c r n}}{\sum_{n=1}^{N}\left(1+a_{n} \mathrm{e}^{\mathrm{j} \phi_{n}}\right)}\right\} \\
& =\Re\left\{\frac{1-\frac{1}{N} \sum_{n=1}^{N} a_{n} \mathrm{e}^{\mathrm{j} \phi_{n}}}{1+\frac{1}{N} \sum_{n=1}^{N} a_{n} \mathrm{e}^{\mathrm{j} \phi_{n}}}\right\} \\
& =\Re\left\{\frac{1-a_{s} \mathrm{e}^{\mathrm{j} \phi_{s}}}{1+a_{s} \mathrm{e}^{\mathrm{j} \phi_{s}}}\right\}
\end{aligned}
$$

where (34) was obtained by making all the jammer baselines equal and

$$
a_{s} \mathrm{e}^{\mathrm{j} \phi_{s}}=\frac{1}{N} \sum_{n=1}^{N} a_{n} \mathrm{e}^{\mathrm{j} \phi_{n}}
$$

is the average return from the non-matched paths through the jammer loops.

Equation (35) shows that the effect of perfectly matched collinear multi-loop retrodirective cross-eye jammer loops with equal baselines is the same as for a single loop. However, the matching between the two paths through the jammer depends on the overall result of the loops rather than of only a single loop. From this observation, it appears that tolerance requirements will be relaxed in the multi-loop case as it is likely that the random variations in each jammer loop will be cancelled by the random variations in the other loops.

As in the concentric case, changing the relative phase of two jammer loops to $180^{\circ}$ dramatically affects the results. Fig. 8 shows the cross-eye gain when the jammerloop amplitudes differ by $0.5 \mathrm{~dB}\left(A_{2} / A_{1}=0.5 \mathrm{~dB}\right)$ and have a $180^{\circ}$ phase difference $\left(\Phi_{2}-\Phi_{1}=180^{\circ}\right)$.

The multi-loop indicated angles in Fig. 8 are significantly larger than the single-loop case. The one exception is the 10-m baseline case, which is identical to the singleloop case as outlined above. Again, this is a result of the fact that the sum-channel returns of the jammer loops cancel, leading to a small sum-channel return, while the difference-channel return remains largely unaffected. This is achieved by exploiting the fact that jammerbaseline ratio $\left(d_{c r n}\right)$ only appears in the numerator of the cross-eye gain in (27). This is the reason that decreasing the smaller baseline $\left(d_{c 1}\right)$ leads to a larger indicated angle as shown in Fig. 8.

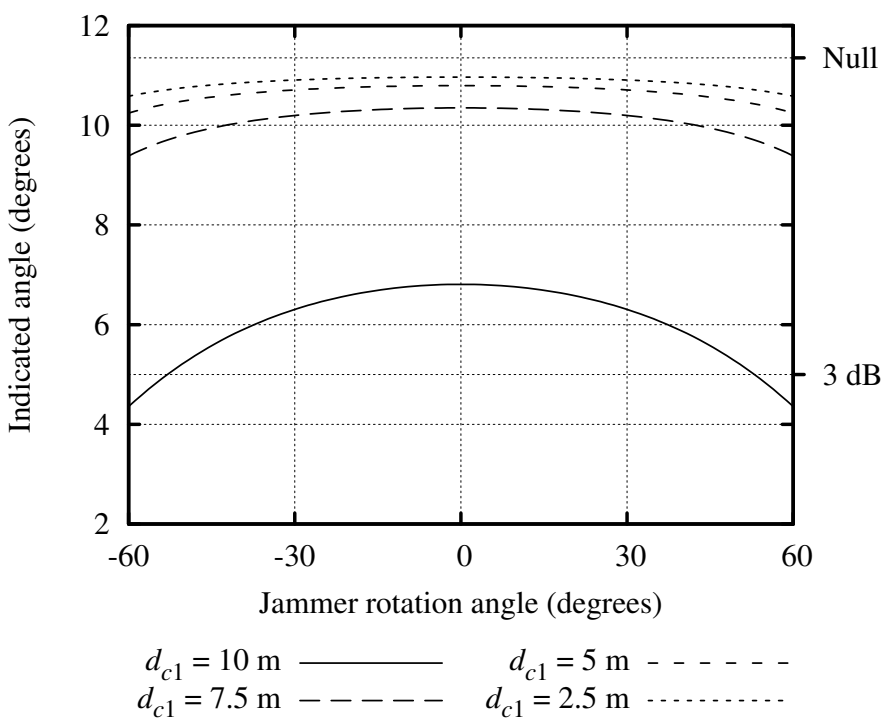

Fig. 8. The indicated angle for identical collinear jammer loops for the case considered in the text.

\section{CONCLUSION}

Previously published analyses of multi-loop retrodirective cross-eye jamming ignored the retrodirective implementation and can thus be expected to have significant errors in this case. The derivation of the cross-eye gain or its equivalent for multi-loop retrodirective crosseye jamming is described, and a number of conclusions based on this analysis are outlined.

Concentric multi-loop retrodirective cross-eye jammers are able to achieve larger indicated angles than the single-loop case by reducing the sum-channel return. However, the fact that the side of the jammer on which the apparent target is generated can vary rapidly for small jammer rotations limits the applicability of this approach. The use of omnidirectional antennas helps somewhat by ensuring a low sum-channel return over all angles, but this low sum-channel return means that this approach is unlikely to be viable in the presence of platform skin return. Alternatively, the sum-channel return can be increased at the expense of lower cross-eye gain to reduce the JSR required by each jammer loop.

Collinear multi-loop retrodirective cross-eye jammers can also achieve larger indicated angles than the singleloop case, again with the caveat that the sum-channel return is significantly reduced. Alternatively, the sumchannel return can be increased as the number of jammer loops increases, thereby reducing the JSR requirements of each loop. Lastly, the fact that the effect of tolerances on the system performance is reduced is demonstrated for some special cases. 


\section{ACKNOWLEDGEMENT}

The author would to express his sincere thanks to the anonymous reviewers for their valuable comments and suggestions.

\section{REFERENCES}

[1] C. Musso and C. Curt, "Robustness of a new angular countermeasure," in Radar 97, 14-16 Oct. 1997, pp. 415-419.

[2] N. M. Harwood, W. N. Dawber, V. A. Kluckers, and G. E. James, "Multiple-element crosseye," IET Radar Sonar Nav., vol. 1, no. 1, pp. 67-73, Feb. 2007.

[3] W. P. du Plessis, "Multi-loop cross-eye jamming," CSIR, Pretoria, R.S.A., Report, 12 Dec. 2012.

[4] S. A. Vakin and L. N. Shustov, "Principles of jamming and electronic reconnaissance - Volume I," U.S. Air Force, Tech. Rep. FTD-MT-24-115-69, AD692642, 1969.

[5] L. B. Van Brunt, Applied ECM. EW Engineering, Inc., 1978, vol. 1.

[6] A. Golden, Radar Electronic Warfare. AIAA Inc., 1987.

[7] D. C. Schleher, Electronic warfare in the information age. Artech House, 1999.

[8] F. Neri, Introduction to Electronic Defense Systems, 2nd ed. SciTech Publishing, 2006.

[9] W. P. du Plessis, J. W. Odendaal, and J. Joubert, "Tolerance analysis of cross-eye jamming systems," IEEE Trans. Aerosp. Electron. Syst., vol. 47, no. 1, pp. 740-745, Jan. 2011.

[10] W. P. du Plessis, "A comprehensive investigation of retrodirective cross-eye jamming," Ph.D. dissertation, University of Pretoria, 2010.

[11] W. P. du Plessis, J. W. Odendaal, and J. Joubert, "Extended analysis of retrodirective cross-eye jamming," IEEE Trans. Antennas Propag., vol. 57, no. 9, pp. 2803-2806, Sept. 2009.
[12] W. P. du Plessis, "Modelling monopulse antenna patterns," in Saudi Int. Electron. Commun. Photon. Conf. (SIECPC), 27-30 April 2013, pp. 1-5.

[13] W. P. du Plessis, "Platform skin return and retrodirective crosseye jamming," IEEE Trans. Aerosp. Electron. Syst., vol. 48, no. 1, pp. 490-501, Jan. 2012.

[14] S. M. Sherman and D. K. Barton, Monopulse principles and techniques, 2nd ed. Artech House, 2011.

[15] W. P. du Plessis, "Limiting apparent target position in skinreturn influenced cross-eye jamming," IEEE Trans. Aerosp. Electron. Syst., vol. 49, no. 3, pp. 2097-2101, July 2013.

[16] W. P. du Plessis, J. W. Odendaal, and J. Joubert, "Experimental simulation of retrodirective cross-eye jamming," IEEE Trans. Aerosp. Electron. Syst., vol. 47, no. 1, pp. 734-740, Jan. 2011.

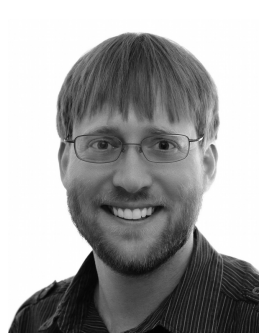

Warren du Plessis (M'00, SM'10) received the B.Eng. (Electronic) and M.Eng. (Electronic) and Ph.D. (Engineering) degrees from the University of Pretoria in 1998, 2003 and 2010 respectively, winning numerous academic awards including the prestigious ViceChancellor and Principal's Medal.

$\mathrm{He}$ spent two years as a lecturer at the University of Pretoria, and then joined Grintek Antennas as a design engineer for almost four years, followed by six years at the Council for Scientific and Industrial Research (CSIR). $\mathrm{He}$ is currently an Associate Professor at the University of Pretoria, and his primary research interests are cross-eye jamming and thinned antenna arrays. 